\title{
PBL 教学在临床医学教育中的应用
}

\author{
王金桥 \\ 军城县中医医院科教科 \\ DOI:10.32629/er.v2i7.1922
}

[摘 要] PBL教学指的是将学习放在复杂、有意义的问题情境中,学生以小组合作形式进行讨论有效地解决其中的问题,这样 学生就能够学习到更多科学知识,提升学生的解决问题能力、自主学习能力 ${ }^{[1]}$ 。因此, 在医学教学过程中, 教师需要引进PBL教 学模式, 提升学生的学习积极性和实践能力。基于此, 文章阐述了 PBL教学的相关内容, 并对PBL教学在临床医学教育中的应用 进行了研究。

[关键词] PBL 教学; 临床医学教育; 应用

\section{引言}

在临床医学专业教学中, 教师通过学习更多的临床知识, 能够提升自身的综合能力, 实现向临床医生进行转变, 在这 一过程中需要加强对学生自主学习能力、发现和解决问题能 力的培养。但是, 在传统的临床医学专业教学过程中, 教师只 注重自身的主体地位, 这种被动的教学模式已无法满足现代 医学对学生的要求。为了有效地改善这一现状, 教师应该实 行以问题为导向 (PBL) 的教学模式, 提高学生终身学习的能 力, 满足现代化医学教育的要求。

\section{$1 \mathrm{PBL}$ 教学的相关内容分析}

PBL教学指的是以学生为中心、教师为引导的教学模式, 大家根据提出的问题, 通过多种方式探究问题背后的原因 和相关知识, 不断培养学生的自主学习能力和终生学习能 力。由于学习目标、教学内容都存在很大的差异, 使得PBL 教学模式的表现方式有所不同, 但教学目标是一致的, 主要 是引导学生进行自主学习, 其核心理念是 “问题为基础, 学 生为主体”, 教师需要转变自身在传统教育模式中的主体地 位, 充分发挥自身的引导作用, 学生通过查阅文献和共同讨 论的方式, 寻找解决问题的方法。在新时期的发展中, PBL 教学模式得到了很多高校的重视, 逐渐发展成医学教育模 式改革的重点内容。

\section{PBL 教学在临床医学教育中的应用优势}

\section{1提高学生的综合素养}

在临床医学教育过程中, 教师需要引进PBL教学模式, 充 分发挥出这种教学模式的作用, 提升学生的自主学习能力和 解决问题的能力, 这样学生就能够在实践和学习过程中, 掌 握更多临床基础知识, 并将其应用到临床实践中。同时, 在分 组式教学、讨论式教学过程中, PBL教学模式的应用使得学生 能够及时发现自身的问题, 并在课余时间利用图书馆、数据 库等方式查阅相关资料, 并在课堂中与学生共同讨论并解决 其中的问题, 培养学生的自主学习能力、分析问题和解决问 题的能力, 激发学生的学习积极性。与传统的教育模式相 比, PBL教学模式具有很大的优势, 在实际教学过程中, 学生 不再是被动地接受知识, 而是积极参与到课堂教学中, 激发
学生的学习积极性, 培养学生的创新能力和创造性思维。另 外, 在PBL教学过程中, 学生的想象力和创造力不会受到影响, 且在实际交流过程中, 学生能够获取不同思维方式中的信息, 明确自身的不足, 看到其他人的优点, 掌握更多的学习内容, 以此为基础合理地调整自己的学习计划和学习目标, 进而提 升学生的创造性思维能力。并且, 在PBL课堂中, 学生通过激 烈的讨论能够提升语言组织能力和人际交往能力, 增强学生 的自信心。

\section{2 提高教师的专业技能和综合素质}

PBL教学模式是以学生为中心的教学模式, 注重学生的 讨论, 有助于教师加强对学生的了解, 并从学生身上学习到 更多优秀的东西, 不断优化教师的教学模式, 进而提升学生 的综合素质和专业教学能力。在PBL课堂教学过程中, 学生占 据着主体位置, 教师要想发挥出自身的引导作用, 需要具备 较强的综合素质, 既要掌握更多专业知识, 还需要将专业知 识应用到实际教学中, 提高教师的逻辑思维能力和综合知识 能力。并且, 在PBL教学过程中, 学生能够发现自己欠缺的内 容, 能够不断扩大知识面, 确保知识储备的全面性。除此之外, 教师应该具备较强的组织领导能力, 合理地把控课堂教学的 时间, 并将课堂时间进行充分利用。因此, 教师需要不断提升 自身的综合素质和专业水平, 实现PBL教学的目标。

\section{3 提高学生的自主学习能力}

在传统的教学过程中, 学生只能够被动地学习知识, 所 有教学方式都需要根据教师的方式进行, 无法发挥出学生自 身的优势, 不利于提升学生的自主学习能力和探索能力。在 PBL教学过程中, 教师需要注重学生的主体地位, 将问题作为 基础, 这样学生就能够通过查阅资料、小组讨论等方式解决 学习中的问题, 使得学生能够主动学习, 激发学生的学习积 极性。除此之外, 与传统的理论知识相比, 医学理论知识相对 比较枯燥, 学生理解存在一定的难度, 无法激发学生的学习 积极性, 这时教师需要引用PBL教学模式, 将一些典型病例融 入实际教学中, 教师针对这些案例提出相关问题, 这样学生就 能够带着问题进行思考和学习, 不断探讨获取解决问题的方 式, 激发学生对学习内容的兴趣, 进而找到解决问题的方法。 
2. 4顺应现代临床医学教学的发展

在PBL教学过程中, 学生不只是知识的被动接受者, 而是 在课堂中占据着主体地位, 有利于激发学生的学习积极性, 充分发挥出学生的主观能动性, 提高学生的自主学习能力。 在现代化社会的发展中, 临床医学教学对学生的创新能力提 出了一定的要求, 而PBL教学的应用有助于激发学生的想象 力和创造力, 培养学生的创新思维, 帮助学生将所学知识应 用到实际案例中, 使得学生将理论知识和临床进行融合, 学 生就能够灵活地应用所学知识, 提高学生的临床思维能力。

\section{PBL 教学模式在临床医学教育应用中注意的问题}

在PBL教学过程中, 教师需要重视临床实践活动, 这样不 仅能够向学生传授更多的医学知识, 还可以使得学生提高学 生的专业水平, 教师在实际教学过程中需要充分发挥自身的 引导作用, 帮助学生在寻找解决方式的过程中不断思考。并 且, 教师应该做好备课工作, 将临床、手术、外科等内容进行 融合, 提高临床医学专业教学效果 ${ }^{[2]}$ 。在新时期的发展 中, PBL教学模式对教师的精力和时间提出了更加严格的要 求, 教师应该提升自身的管理能力, 提出有针对性的问题。现 阶段, PBL教学模式的应用还缺乏一定的规范性, 教育部门未 针对临床医学教育制定规范的教材, 缺乏相应的考核方式, 且学生的学习积极性、教师选择的课堂教学内容和辅导方式 都会对PBL教学效果带来一定的影响。

\section{PBL 教学在临床医学教育中的应用}

4. 1 充分发挥学生在PBL教学中的主体地位

在PBL教学改革过程中, 很多学生无法接受这种教学模 式, 且无法适应新型的教学模式, 使得学生失去了学习的信 息, 这时教师可以应用 “一带一帮” 的教学模式, 鼓励学生积 极参与到实际教学过程中。除此之外, 在PBL教学过程中, 教 师需要让学生充分理解并信任这种教学模式, 在教学初期学 校可以组织学生参加相关讲座活动, 讲述PBL教学的成功案 例, 总结PBL教学中的成果和成功案例, 这样学生就能够感受 到PBL教学改革带来的好处, 激发学生的学习积极性。
4.2 建立完善的PBL教学队伍

在临床医学教育教学过程中, 教师应该重视学生的心理 活动, 在课前给予学生更多的心理暗示, 使得学生能够做好 准备, 并在实际教学过程中加强和教师之间的交流和沟通。 PBL教学模式在临床医学教育中的应用, 对教师的基本素质 提出了更高的要求, 教师需要转变自身的观念, 加强对PBL教 学模式的重视。除此之外, 学校应该加大PBL教学师资队伍建 设力度, 引进更多具备PBL教学经验的教师参与到实际教学 过程中, 并安排教师参加相关培训活动, 使得教师树立先进 的教学理念, 实现PBL教学的效果。在经典的PBL教学过程中, 教师普遍使用小组教学方式, 这就需要更多优秀的教师, 但 我国师资和教学资源相对匮乏, 影响着PBL小组教学模式的 应用效果。但是, 在新时期的发展中, PBL教学模式不受时间 和空间的限制, 教师和学生可以在线进行PBL教学, 增强学生 之间的交流和沟通, 改善教师分配不均匀的问题, 实现PBL教 学模式的有效实施和发展, 为我国医学教育提供一个新的选 择 ${ }^{[3]}$ 。

\section{5 结束语}

综上所述, 在现代化社会的发展中, 社会各个行业对优 秀人才的需求量日益增加, 这就需要教师创新并优化传统的 教学模式。在临床医学专业教学过程中, 传统的教学模式已 无法满足新时期临床教学的实际需求, 教师需引进PBL教学 模式, 以满足医学人才培养的实际需求。并且, 在教育行业的 发展中, 相关部门需要不断完善PBL教学模式, 将其应用到更 多领域教学过程中, 为社会发展培养出更多优秀人才。

\section{[参考文献]}

[1]张英杰.PBL教学模式在我国医学教育中的应用研究 [J].世界最新医学信息文摘,2018,18(70):280-281.

[2]邵子月,陈否,史淲悦,等.PBL教学法在临床医学教学 中的实践剖析[J]. 医学理论与实践,2018,31(15):2361-2363.

[3]杨金华.浅析 PBL 教学法在临床医学教学中的不足[J]. 长治学院学报,2018,35(02):103-105. 